\title{
Lyse Roy et Yves Gingras, dir. Les Universités nouvelles. Enjeux et perspectives
}

\author{
Québec: Presses de I'Université du Québec, 2012. 342 pages.
}

\section{Micheline Cambron}

Université de Montréal

Résultat d'un colloque organisé à l'occasion du 40ªnniversaire de l'UQAM, l'ouvrage collectif dirigé par Lise Roy et Yves Gingras porte sur le phénomène qu'a constitué l'émergence des universités " nouvelles ", lesquelles cherchaient à mettre en œuvre des modèles académiques, urbanistiques et administratifs éloignés des pratiques traditionnelles. Le résultat est aussi instructif que stimulant. Les analyses, solidement documentées, éclairent des expériences menées dans différents pays et la perspective historique adoptée révèle les enjeux cruciaux, les écueils, les points aveugles, tout en dégageant à grands traits l'imagination éducative à l'œuvre dans le dernier demisiècle. Prises dans leur ensemble, les contributions se révèlent ainsi un puissant outil d'analyse des pratiques universitaires et des idéaux qui les meuvent.

Toutes les contributions illustrent les relations étroites qui existent entre valeurs académiques, usages politiques, pratiques de gestion, partages disciplinaires, choix architecturaux et urbanistiques. Ainsi, lorsque Marcel Fournier et Dan Antonat racontent les péripéties entourant la construction de l'UQAM, nous pouvons observer les divers agents à l'œuvre, le choc entre les valeurs affichées et le réel, les compromis adoptés. Le même type d'observations se retrouve à propos du développement des universités nouvelles au Canada anglophone, sous la plume de Maurice Lagueux, qui insiste sur le rôle joué par les signatures architecturales dans le développement d'un sentiment d'appartenance. Ces deux textes mériteraient à eux seuls le détour parce qu'ils nous révèlent les effets sociétaux larges que la création d'universités charrie et notre profonde ignorance de l'histoire de nos universités. On se prend à rêver d'une histoire générale des universités québécoises qui, loin des hagiographies, raconterait les luttes diverses qui ont fait nos institutions, sans négliger leurs rendez-vous ratés avec les communautés qui les portent. Étudiée par François Hiraux, l'expérience de l'Université de Louvain témoigne aussi de la force des liens entre collectivité et université lorsque ceux-ci se déploient non seulement au niveau académique mais aussi 
aux plans identitaire et territorial. Favorables au développement de l'Université ces liens peuvent cependant devenir funestes aux idéaux humanistes originels, passés à la moulinette de la rentabilité sociale. Il semblerait ainsi que le "nouveau " soit plus fragile, moins capable de résister aux pressions externes. Le cas de l'École supérieure technique Bauman de Moscou étudié par Carole Sigman en est une illustration, l'autonomie universitaire revendiquée y ayant servi d'écran à l'aliénation de la recherche, dans le déni des spécificités universitaires.

Les liens entre les universités et les milieux qui les portent sont abordés également à propos des enseignements techniques. Yves-Claude Lequin et Pierre Lamard examinent le développement de l'enseignement technique en France entre 1968 et 2010, s'attardant à la création de cursus visant l'augmentation du nombre d'ingénieurs et de techniciens de haut niveau. L'implication des milieux dans le développement universitaire régional, les difficultés d'ordre administratif, les lenteurs et les incohérences des décisions gouvernementales, les conflits entre disciplines : tout témoigne du poids que font peser sur la création des institutions des pratiques de gestion réputées neutres. L'ordre aveugle que constitue la gestion est aussi un agent important dans le récit que fait Virginie Fonteneau des initiatives visant à créer la Faculté des sciences et techniques de Nantes. S'y oppose la dimension humaine, seule susceptible de favoriser la conciliation entre « le monde des savoirs et le monde de l'action » (173), principal enjeu du développement d'un enseignement technique. Hocine Kelfaoui analyse quant à lui le cas des instituts technologiques algériens et révèle les conflits souterrains qui opposent les logiques théorique et pratique dans leur quête respective d'identité, de responsabilité et de pouvoir, illustrant ainsi la force d'inertie des modèles académiques anciens et des habitus institutionnels, même en contexte de "nouveauté ".

Plusieurs textes abordent la question de la pédagogie nouvelle qui serait propre aux universités " nouvelles ». Le récit que fait Charles Soulié du développement du Centre universitaire expérimental de Vincennes n'est guère édifiant : grenouillages, querelles d'égo, impérialisme disciplinaire. La pédagogie nouvelle semble avoir camouflé là une entreprise de réorganisation hiérarchique des savoirs très éloignée des objectifs d'interdisciplinarité et de développement collaboratif des savoirs. D'autres textes abordent frontalement la question des valeurs pédagogiques. Paul Bitsh et Lorna Heaton identifient trois des objectifs centraux de l'université nouvelle — pédagogie interactive plutôt que magistrale, accent mis sur la pertinence sociétale et interdisciplinarité - et montrent comment la Roskilde University (Danemark) a pu demeurer fidèle à ces objectifs grâce à divers accommodements. Le processus de Bologne apparaît alors comme le choc qui révèle le dilemme entre processus collectif d'apprentissage et objectifs individuels de formation. Le même dilemme se trouve au cœur de la création de l'ENAP, dont Caroline Dufour retrace l'histoire en soulignant les tensions entre vision étroitement instrumentalisée de la formation et idéaux humanistes, l'interdisciplinarité s'y révélant finalement un écueil. Une chromatographie du prestige académique, de Michel Jedrak, ne convainc guère mais témoigne aussi des possibles dérives gestionnaires liées aux abus du marketing.

Pourtant, il serait injuste de prétendre que les idéaux des universités nouvelles ne sont pas créateurs. Si Denis Bertrand, qui traite de la création de l'UQAM, montre 
comment plusieurs problèmes — sous-évaluation des effectifs étudiants, sous-évaluation de la force des attachements disciplinaires et institutionnels antérieurs, complexité des relations avec le gouvernement et complications de la gestion des chantiers — ont mis à l'épreuve les idéaux initiaux identifiés par Claude Corbo dans le texte liminaire - accessibilité et démocratisation; renouvellement des pratiques pédagogiques; mise à contribution de la formation à distance; résistance à l'homogénéisation des pratiques; capacité de changement ancrée dans une identité institutionnelle forte (22) - le texte de Sylvie Bonin, Johanne Bujold et Pierre Doray sur la transformation des populations étudiantes fait la démonstration des effets positifs liés à la création des universités " nouvelles " au Québec : augmentation de la diplomation, particulièrement chez les femmes des régions urbaines, et transformation des clientèles (âge, origine sociale, capital scolaire familial). Les différents tableaux qui montrent l'évolution des populations étudiantes de première génération sont éloquents et invitent à une analyse de l'écologie des universités québécoises. En effet, les universités nouvelles ne vivent pas en vase clos. Une initiative comme celle de TELUQ, dont l'histoire mouvementée et quasi feuilletonnesque est analysée par Patrick Guillemet gagnerait ainsi à être replacée dans l'histoire plus générale des initiatives antérieures en ce sens. Qui se souvient de l'Heure provinciale (1929-1939), placée sous l'égide de Université de Montréal? Les utopies universitaires de démocratisation des savoirs ne sont pas apparues au tournant des années 60. Elles datent du Moyen âge au moins, même si les universités nouvelles les portent de manière plus explicite et plus militante, avec tous les avantages et tous les risques que cela comporte.

Cet ouvrage nous invite à interroger l'histoire des universités, leur mission, et les relations toujours compromises avec la société qui marquent la quête collective du savoir. Nous en avons le plus urgent besoin. 